Moroccan J. of Pure and Appl. Anal. (MJPAA)

Volume 5(2), 2019, Pages 251-262

ISSN: Online 2351-8227 - Print 2605-6364

DOI 10.2478/mjpaa-2019-0018

\title{
Boyd and Wong Type Fixed Point Theorems in Partial Metric Spaces
}

\author{
FAUstine NzIKU ${ }^{1}$ AND SANTOSH KUMAR ${ }^{2}$
}

\begin{abstract}
Aвstract. In this paper, we present fixed point results for Boyd and Wong type [3] generalized contractive condition in partial metric spaces. In particular, we generalize the fixed point results due to Akkouchi [1] in complete partial metric spaces in which the continuity requirement for a mapping is relaxed to obtain the results. In addition to that we present a common fixed point theorem for a pair of maps. An illustrative example is also constructed to exhibit the results.
\end{abstract}

Mathematics Subject Classification (2010). 47H10, 54H25.

Key words and phrases. Metric spaces, Partial metric spaces, Fixed point, Boyd and Wong contractive condition.

\section{Introduction and Preliminaries}

Banach fixed-point theorem is an important tool in the theory of metric spaces. It guarantees the existence and uniqueness of fixed points of certain self-maps of metric spaces, and provides a constructive method to find those fixed points. It has been extensively studied by many researchers to address different problems in analysis. This theorem [2] states that, if $X$ is a non-empty set and $d$ is a metric on $X$ such that, $d: X \times X \rightarrow[0, \infty]$ and if $T$ is a self mapping on a complete metric space $(X, d)$ which satisfies:

$$
d(T x, T y) \leq c d(x, y)
$$

Received 05 November 2019 - Accepted: 21 December 2019.

(C) The Author(s) 2017. This article is published with open access by Sidi Mohamed Ben Abdallah University.

1,2 Depertment of Mathematics, College of Natural and Applied Sciences, University of Dar es Salaam, Tanzania e-mail: ${ }^{1}$ faustinenziku@gmail.com

e-mail: ${ }^{2}$ drsengar2002@gmail.com . 
for some $0 \leq c<1$ for all $x, y \in X$, then $T$ has a unique fixed point $x_{0}$ and a sequence $\left\{T^{n} x\right\}$ converges to $x_{0}$ for $x \in X$.

Boyd and Wong [3] gave an important generalization of this theorem due to Banach [2] in metric spaces. The theorem states as follows:

Theorem 1. [3] Let $(M, d)$ be a complete metric space and let $P:=\{d(x, y): x, y \in M\}$. Let $T: M \rightarrow M$ be a self mapping satisfying:

$$
d(T x, T y) \leq \chi(d(x, y)), \forall x, y \in M,
$$

where $\chi: \bar{P} \longmapsto[0, \infty)$ is upper semi continuous from the right on $\bar{P}$ and satisfies $\chi(t)<t, \forall$ $t \in \bar{P} \backslash\{0\},(\bar{P}$ denotes the closure of $P)$. Then $T$ has a unique fixed point $z$ and $d\left(T^{n} x, z\right) \rightarrow 0, \forall$ $x \in M$.

Rakotch [9] proved that if $\alpha(t)$ is a decreasing function where $t=d(x, y)$, with the property that $\alpha(t)<1$ for $t>0$, then the mapping $T$ satisfying (1.2) by taking $\chi(t)=\alpha(t) t$ has a unique fixed point. It is easy to show that if $\alpha(t)$ is an increasing function and $\alpha(t)<1$ for $t \geq 0$, Banach's Contraction Principle (BCP) still holds. Boyd and Wong proved that the only requirement to hold the $\mathrm{BCP}$ is that $\chi(t)$ to be semi-continuous and $\chi(t)<t$ for $t>0$.

Akkouchi [1] observed that the contractive condition (1.2) above signifies that $T$ must be continuous. He generalized this condition in such a way that no information on continuity of $T$ is needed to derive the results.

Matthews [7] introduced partial metric spaces as the foundation to study the denotational semantics of programming languages whereby a non-Hausdorff topological model is constructed as a system of logic for a programming language. Partial metric is a metric based tools for program verification in which the notion of the size of a data object in a domain is used in quantifying how the data object is well defined in the domain.

The following definitions are due to Matthews [7].

Definition 1.1. [7] Let $X$ be a non-empty set. A function $p: X \times X \rightarrow[0, \infty)$ is called a partial metric on $X$ if it satisfies the followings:

(P0): $0 \leq p(x, x) \leq p(x, y)$ (non-negativity and small self-distance).

(P1): $p(x, y)=p(x, x)=p(y, y) \Rightarrow x=y$ (indistancy implies equality).

(P2): $p(x, y)=p(y, x)$ (symmetric).

(P3): $p(x, y) \leq p(x, z)+p(z, y)-p(z, z)$ (triangularity), for all $x, y, z \in X$.

A pair $(X, p)$ is called a partial metric space.

Note that $p(x, y)=0$ implies $x=y$ by $(P 0-P 1)$. In general the converse is not true. Therefore, a metric space is a partial metric space with all self-distances zero.

In computer science applications $p(x, x)$ referred to as the size or weight of $x$. This quantity structure is used to describe the amount of information contained in $x$. We say that the data object $x$ is more defined if the value of $p(x, x)$ is smaller and it is totally defined if $p(x, x)=0$.

Definition 1.2. [7] Let $\left\{x_{n}\right\}$ be a sequence in a partial metric space $(X, p)$, then,

(i) A sequence $\left\{x_{n}\right\}$ is said to be convergent to a point $x \in X$ if and only if $p(x, x)=$ $\lim _{n \rightarrow \infty} p\left(x, x_{n}\right)=\lim _{n \rightarrow \infty} p\left(x_{n}, x_{n}\right)$. 
(ii) A sequence $\left\{x_{n}\right\}$ is called a Cauchy sequence if there exists $a \geq 0$ such that for each $\epsilon>0$ there exists $N$ such that for all $n, m>N,\left|p\left(x_{n}, x_{m}\right)-a\right|<\epsilon$.

In other words, the sequence $\left\{x_{n}\right\}$ is Cauchy if the numbers $p\left(x_{n}, x_{m}\right)$ converges to some $a$ as $n, m$ approach infinity, that is, if $\lim _{n, m \rightarrow+\infty} p\left(x_{n}, x_{m}\right)=a$, where $a$ is a finite number.

(iii) A partial metric space $(X, p)$ is complete if every Cauchy sequence $\left\{x_{n}\right\}$ converges to a point $x \in X$ such that $p(x, x)=\lim _{n, m \rightarrow+\infty} p\left(x_{n}, x_{m}\right)$.

Definition 1.3. [7] A contraction on a partial metric space $X$ is a function $f: X \rightarrow X$ such that there exist a constant $0 \leq c<1$ for all $x, y \in X$ satisfies that;

$$
p(f(x), f(y)) \leq c(p(x, y)) .
$$

The following lemma is due to Matthews [7],

Lemma 2. [7] Let $X$ be a non-empty set and $p$ be a partial metric on $X$. Then, the function $p^{s}$ : $X \times X \rightarrow[0, \infty)$ defined as,

$$
p^{s}(x, y)=2 p(x, y)-p(x, x)-p(y, y)
$$

for all $x, y \in X$ is a metric on $X$.

We take note that, in a partial metric space $(X, p)$, a convergent sequence may not be a Cauchy sequence. For instance consider Example (1.4) in the work of Nazir and Abbas [8].

The following definition was given by Akkouchi [1].

Definition 1.4. [1] Let $\Phi$ be the set of continuous functions $\phi:[0, \infty) \rightarrow[0, \infty)$ satisfying,

(i) $\phi(t)=0$ if and only if $t=0$ and

(ii) For all sequences $\left\{t_{n}\right\}$ of elements in $[0, \infty)$, if $\left\{\phi\left(t_{n}\right)\right\}$ is a decreasing sequence then the sequence $\left\{t_{n}\right\}$ is bounded, that is $\sup _{n}\left(t_{n}\right)<\infty$.

We observe that if $\phi:[0, \infty) \rightarrow[0, \infty)$ is a continuous function satisfying either of the following then it must belong to $\Phi$;

(iii) $\phi$ is non decreasing in $[0, \infty)$

(iv) $\phi(t) \geq M t^{a}$ for all $t>0$ where $M, a>0$.

The following theorem is due to Akkouchi [1];

Theorem 3. [1] Let $(M, d)$ be a complete metric space and let $P:=\{d(x, y): x, y \in M\}$. Let $\phi \in \Phi$ and $\chi: \overline{\phi(P)} \rightarrow[0, \infty)$ be an upper semi continuous from the right on $\overline{\phi(P)}$ and satisfies $\chi(t)<t$ for all $t \in \overline{\phi(P)} \backslash\{0\}$ where $\overline{\phi(P)}$ denotes the closure of $\phi(P)$. Let $T$ be the mapping of $M$ satisfying the following contractive condition,

$$
\phi(d(T x, T y)) \leq \chi(\phi(d(x, y))), \forall x, y \in M .
$$

Then $T$ has a unique fixed point $z$ and $d\left(T^{n} x, z\right) \rightarrow 0$ for all $x \in M$ as $n \rightarrow \infty$. 


\section{Main Results}

We now state and prove the generalization of Theorem 3 and then provide an illustrative example thereafter.

Theorem 4. Let $(X, p)$ be a complete partial metric space and let $P:=\{p(x, y): x, y \in X\}$. Let $\phi \in \Phi$ and $\chi: \overline{\phi(P)} \rightarrow[0, \infty)$ be an upper semi continuous from the right on $\overline{\phi(P)}$ and let $\chi(t)<t$ for all $t \in \overline{\phi(P)} \backslash\{0\}$ where $\overline{\phi(P)}$ denotes the closure of $\phi(P)$. Let $T$ be a self mapping of $X$ such that

$$
\phi(p(T x, T y)) \leq \chi(\phi(p(x, y))) \text { for all } x, y \in X .
$$

Then $T$ has a unique fixed point $z$ such that $T z=z$ where $p(z, z)=0$.

Proof. (a) First we will construct a sequence of points $x_{1}, x_{2}, x_{3}, \ldots$ as follows:

Let a point $x_{0} \in X$, and set $x_{n}=T x_{n-1}$ for all integers $n \geq 1$.

Also consider, $a_{n}=p\left(x_{n}, x_{n+1}\right)$ for all $x_{n} \in X$ and all integers $n \geq 0$.

For all $n \geq 0$ we have,

$$
\phi\left(a_{n+1}\right)=\phi\left(p\left(x_{n+1}, x_{n+2}\right)\right) .
$$

By considering (2.1) we can have,

$$
\phi\left(p\left(x_{n+1}, x_{n+2}\right)\right)=\phi\left(p\left(T x_{n}, T x_{n+1}\right)\right) \leq \chi\left(\phi\left(p\left(x_{n}, x_{n+1}\right)\right)\right) .
$$

Hence, $\phi\left(a_{n+1}\right) \leq \chi\left(\phi\left(a_{n}\right)\right) \leq \phi\left(a_{n}\right)$, for all integers $n \geq 0$.

This implies that $\left\{\phi\left(a_{n}\right)\right\}$ is a decreasing sequence for all integers $n \geq 0$.

Now consider, $a$ be the limit point for the sequence $\left\{\phi\left(a_{n}\right)\right\}$, it is obvious that $a \in[0, \infty)$. We claim that $a=0$. Suppose in contrary that $a>0$, then from (2.1) we have,

$$
\phi\left(p\left(x_{n+1}, x_{n+2}\right)\right)=\phi\left(p\left(T x_{n}, T x_{n+1}\right)\right) \leq \chi\left(\phi\left(p\left(x_{n}, x_{n+1}\right)\right)\right) .
$$

Thus,

$$
\phi\left(a_{n+1}\right) \leq \chi\left(\phi\left(a_{n}\right)\right)
$$

so that;

$$
0<a \leq \limsup _{n \rightarrow \infty} \chi\left(\phi\left(a_{n}\right)\right) \leq \chi(a),
$$

which is a contradiction on the assumption of the function $\chi$, hence

$$
\lim _{n \rightarrow \infty} \phi\left(a_{n}\right)=a=0 .
$$

Since the sequence $\left\{\phi\left(a_{n}\right)\right\}$ is decreasing and satisfies (ii) of Definition 1.4 such that $\lim _{n \rightarrow \infty} a_{n}<$ $\infty$, then the sequence $\left\{a_{n}\right\}$ is bounded for all integers $n \geq 0$.

Since the sequence $\left\{a_{n}\right\}$ is bounded, then it contains a convergent subsequence say $\left\{a_{n_{k}}\right\}$ such that $\lim _{k \rightarrow \infty} a_{n_{k}}=b$.

Since $\phi$ is continuous it follows that,

$$
\lim _{k \rightarrow \infty} \phi\left(a_{n_{k}}\right)=\phi(b)=0 .
$$


From $(i)$ of Definition 1.4 we have, $b=0$.

Since we choose the sequence $\left\{a_{n_{k}}\right\}$ arbitrarily, then we can conclude that any convergent subsequence $\left\{a_{n_{k}}\right\}$ of the sequence $\left\{a_{n}\right\}$ converges to zero, hence,

$$
\lim _{n \rightarrow \infty} a_{n}=0 .
$$

We have proved that the sequence $\left\{a_{n}\right\}$ is bounded and converges to zero.

(b) Now consider the sequence $\left\{x_{n}\right\} \in X$. We want to show that the sequence $\left\{x_{n}\right\}$ is a Cauchy sequence.

In contrary suppose that $\left\{x_{n}\right\}$ is not a Cauchy sequence, therefore, $\exists \epsilon>0$ and sequences of integers $\{m(r)\},\{n(r)\}$ such that;

$$
p\left(x_{n(r)}, x_{m(r)}\right) \geq \epsilon
$$

for all $n(r)>m(r) \geq r$ for some $r \geq 0$.

Moreover, suppose that $m(r)$ is chosen as the smallest integer in such a way that inequality (2.5) above holds so that we have,

$$
p\left(x_{n(r)}, x_{m(r)-1}\right)<\epsilon
$$

Now for all positive integers $n(r)>m(r)$ we have the following triangle inequality;

$$
\begin{aligned}
P_{r} & =p\left(x_{n(r)}, x_{m(r)}\right) \\
& \leq p\left(x_{n(r)}, x_{m(r)-1}\right)+p\left(x_{m(r)-1}, x_{m(r)}\right)-p\left(x_{m(r)-1}, x_{m(r)-1}\right) \\
& \leq p\left(x_{n(r)}, x_{m(r)-1}\right)+p\left(x_{m(r)-1}, x_{m(r)}\right) .
\end{aligned}
$$

Considering (2.6) we obtain,

$$
P_{r}=p\left(x_{n(r)}, x_{m(r)}\right) \leq \epsilon+a_{m(r)} .
$$

As $r \rightarrow \infty$ in (2.8) and considering (2.4) we see that $a_{m(r)} \rightarrow 0$, hence, $P_{r} \rightarrow \epsilon$.

But now, for all positive integers $n(r)>m(r)$ we have,

$$
\begin{aligned}
P_{r} & =p\left(x_{n(r)}, x_{m(r)}\right) \\
& \leq p\left(x_{n(r)}, x_{n(r)+1}\right)+p\left(x_{n(r)+1}, x_{m(r)+1}\right)+p\left(x_{m(r)+1}, x_{m(r)}\right) \\
& -p\left(x_{n(r)+1}, x_{n(r)+1}\right) \\
& \leq p\left(x_{n(r)}, x_{n(r)+1}\right)+p\left(x_{n(r)+1}, x_{m(r)+1}\right)+p\left(x_{m(r)+1}, x_{m(r)}\right) .
\end{aligned}
$$

Now,

$$
\begin{aligned}
P_{r}=p\left(x_{n(r)}, x_{m(r)}\right) & \leq a_{n(r)}+p\left(x_{n(r)+1}, x_{m(r)+1}\right)+a_{m(r)} \\
& \leq a_{n(r)}+a_{m(r)}+\chi\left(p\left(x_{n(r)+1}, x_{m(r)+1}\right)\right) .
\end{aligned}
$$

As $r \rightarrow \infty$ in (2.10) and considering (2.4) we see that $a_{m(r)} \rightarrow 0$ and $a_{n(r)} \rightarrow 0$, hence we obtain, $\epsilon \leq \chi(\epsilon)$, which is a contradiction for $\epsilon>0$.

Therefore, the sequence $\left\{x_{n}\right\}$ is a Cauchy sequence. Consequently,

$$
\lim _{n, m \rightarrow \infty} p\left(x_{n}, x_{m}\right)=0 .
$$


(c) Since $X$ is complete then we can choose a point $z \in X$ such that $z$ is the limit of the sequence $\left\{x_{n}\right\}$ and $\lim _{n \rightarrow \infty} p\left(x_{n}, z\right)=0$. Now we prove that $z$ is the fixed point of $T$ and $p(z, z)=$ 0 .

By considering (2.1), for all integers $n \geq 0$ we have,

$$
\phi\left(p\left(x_{n+1}, T z\right)\right)=\phi\left(p\left(T x_{n}, T z\right)\right) \leq \chi\left(\phi\left(p\left(x_{n}, z\right)\right)\right.
$$

Letting $n \rightarrow \infty$ in (2.12) above we get;

$$
\begin{aligned}
\phi(p(z, T z)) & =\lim _{n \rightarrow \infty} \phi\left(p\left(x_{n+1}, T z\right)\right) \\
& \leq \limsup _{n \rightarrow \infty} \chi\left(\phi\left(p\left(x_{n}, z\right)\right)\right. \\
& =\chi(\phi(0)) \\
& \leq \phi(0)=0 .
\end{aligned}
$$

From (2.13) above we have,

$$
\phi(p(z, T z))=0 .
$$

By using $(i)$ of Definition 1.4 we see that,

$$
p(z, T z)=0 .
$$

Hence by $(P 0)$ and $(P 1)$ we conclude that $z=T z$, hence $z$ is a fixed point of $T$ and $p(z, z)=0$.

(d) We now show that $z$ is the unique fixed point of $T$.

Suppose that there exists another fixed point $z_{0} \neq z$ of $T$.

From our contractive condition (2.1), we have,

$$
\begin{aligned}
0<\phi\left(p\left(z_{0}, z\right)\right) & =\phi\left(p\left(T z_{0}, T z\right)\right) \\
& \leq \chi\left(\phi\left(p\left(z_{0}, z\right)\right)\right) \\
& <\phi\left(p\left(z_{0}, z\right)\right)
\end{aligned}
$$

which is a contradiction, hence $z$ is a unique fixed point of $T$.

Remark 5. By setting the function $\phi$ to be the identity mapping in Theorem 4 , then (2.1) which is,

becomes,

$$
\phi(p(T x, T y)) \leq \chi(\phi(p(x, y)))
$$

$$
p(T x, T y) \leq \chi(p(x, y)),
$$

which leads to the following corollary:

Corollary 6. Let $(X, p)$ is a complete partial metric space. Let $\chi:[0, \infty) \rightarrow[0, \infty)$ be an upper semi continuous mapping from the right on $[0, \infty)$ such that $\chi(t)<t$ for all $t>0$. Let $T: X \rightarrow X$ be $a$ mapping satisfying,

$$
p(T x, T y) \leq \chi(p(x, y))
$$

for all $x, y \in X$. Then there exists a unique fixed point $x_{0} \in X$ for mapping $T$ where $p\left(x_{0}, x_{0}\right)=0$. 
We observe that, Corollary 6 above is the generalization of Boyd and Wong [3] fixed point theorem in partial metric spaces which is the work of Romaguera [10].

Remark 7. By setting the function $\phi$ to be an identity mapping in Theorem 4 and letting $\chi(\alpha)=k \alpha$ for $k \in[0,1)$ and $\alpha \geq 0$, then (2.1) which is,

$$
\phi(p(T x, T y)) \leq \chi(\phi(p(x, y)))
$$

becomes,

which leads to the following corollary:

$$
p(T x, T y) \leq k p(x, y)
$$

Corollary 8. Let $(X, p)$ be a complete partial metric space and let $T: X \rightarrow X$ be a mapping such that,

$$
p(T x, T y) \leq k p(x, y)
$$

for all $x, y \in X$ and $k \in[0,1)$. Then $T$ has a unique fixed point $z$ such that $T z=z$ and $p(z, z)=0$.

Which is the Banach fixed point theorem in partial metric space by Matthews [7].

Now, we present an example to show that our results improves the existing results in the literature as there is no any information given on the continuity of mapping $T$ in the process of deriving the results.

Example 2.1. Let $X=[0, \infty)$ be a set with partial metric $p: X \times X \rightarrow[0, \infty)$ which is defined by,

$$
p(x, y)=\max \{x, y\}=t
$$

for all $x, y \in X$.

Using $(P 0-P 3)$ it is easy to check that $(X, p)$ is partial metric space. It is easy to check that the space $(X, p)$ is a complete partial metric space. In fact $p^{*}$ from Lemma 2 is the Euclidean metric on $X$.

Define,

$$
T x=\left\{\begin{array}{cc}
x-\frac{1}{4} x^{2}, & x \in[0,1) \\
x-1, & x \in[1, \infty) .
\end{array}\right.
$$

Also we define,

$$
\phi(t)=\left\{\begin{array}{cc}
t-\frac{1}{2} t^{2}, & t \in[0,1), \\
t-\frac{1}{2}, & t \in[1, \infty) .
\end{array}\right.
$$

We can observe that, $\phi(t)$ is a continuous and non decreasing function, then from Definition 1.4, $\phi$ belongs to $\Phi$.

Thus for all $x, y \in[0,1)$ we have,

$$
\begin{aligned}
\phi(p(T x, T y)) & =\phi(\max \{T x, T y\}) \\
& =\phi\left(\max \left\{x-\frac{1}{4} x^{2}, y-\frac{1}{4} y^{2}\right\}\right) \\
& \leq \phi(\max \{x, y\}) \\
& =\phi(p(x, y)) \\
& =\phi(t)=t-\frac{1}{2} t^{2} .
\end{aligned}
$$


Similarly for $x, y \in[1, \infty)$ we have,

$$
\begin{aligned}
\phi(p((T x, T y))) & =\phi(\max \{T x, T y\}) \\
& =\phi(\max \{x-1, y-1\}) \\
& \leq \phi(\max \{x, y\}) \\
& =\phi(p(x, y)) \\
& =\phi(t)=t-\frac{1}{2} .
\end{aligned}
$$

Thus if we define our mapping $\chi$ by,

$$
\chi(\phi(t))=\left\{\begin{array}{cc}
t-\frac{1}{2} t^{2}, & t \in[0,1) \\
t-\frac{1}{4}, & t \in[1, \infty) .
\end{array}\right.
$$

Then $\chi(\phi(t))$ is upper semi-continuous from right on $[0, \infty)=\overline{\phi(P)}, \chi(t)<t$, for all $t \in$ $\overline{\phi(P)} \backslash\{0\}$ and condition (2.1) holds. Therefore our mapping $T$ has a unique fixed point $x=0$ such that $T(0)=0$ and $p(0,0)=\max \{0,0\}=0$.

In the following theorem we obtain a unique common fixed point for a pair of maps by using generalized Boyd and Wong type contractive condition on complete partial metric spaces. This result is the consequence of Theorem 4, besides here we employ a pair of maps instead of one map.

Before stating our theorem, we give the following lemma which results from Definition 1.1.

Lemma 9. Let $(X, p)$ be a complete partial metric space,

(i) If $p(x, y)=0$, then $x=y$.

(ii) If $x \neq y$, then $p(x, y)>0$.

Note that the proof of the above Lemma follows from Definition 1.1.

Theorem 10. Let $(X, p)$ be a complete partial metric space and let $P:=\{p(x, y): x, y \in X\}$. Let $\phi \in \Phi$ and $\chi: \overline{\phi(P)} \rightarrow[0, \infty)$ be an upper semi continuous from the right on $\overline{\phi(P)}$ and let $\chi(t)<t$ for all $t \in \overline{\phi(P)} \backslash\{0\}$ where $\overline{\phi(P)}$ denotes the closure of $\phi(P)$. Let $T$ and $F$ be self mappings of $X$ such that:

$$
\phi(p(T x, F y)) \leq \chi(\phi(S(x, y))),
$$

for all $x, y \in X$, where $S(x, y)=\max \left\{p(x, y), p(x, T x), p(y, F y), \frac{p(x, F y)+p(y, T x)}{2}\right\}$. Then $T$ and $F$ have a unique common fixed point $z \in X$ such that Fix $T=$ Fix $F=\{z\}$.

Proof. Let $x_{0} \in X$. Define a sequence $\left\{x_{n}\right\}$ by $x_{n+1}=T x_{n}$ and $x_{n+2}=F x_{n+1}$, for all $n=$ $0,1,2,3, \ldots$.

Now, if there exists a positive integer $N$ such that, $x_{N}=x_{N+1}$, then $x_{N}$ is a fixed point of $T$. Similarly, if there exists an integer $n \geq 0$ such that $x_{n+1}=x_{n+2}$, then $x_{n+1}$ is a fixed point of $F$. Here, we suppose that $x_{n} \neq x_{n+1}$, for all integers $n \geq 0$. Let $a_{n}=p\left(x_{n}, x_{n+1}\right)$, for all $\left\{x_{n}\right\} \in X$. For all integers $n \geq 0, \phi\left(a_{n+1}\right)=\phi\left(p\left(x_{n+1}, x_{n+2}\right)\right)$. Considering (2.18) we have,

$$
\begin{aligned}
& \phi\left(a_{n+1}\right) \\
= & \phi\left(p\left(x_{n+1}, x_{n+2}\right)\right)=\phi\left(p\left(T x_{n}, F x_{n+1}\right)\right) \leq \chi\left(\phi\left(S\left(x_{n}, x_{n+1}\right)\right)\right)
\end{aligned}
$$




$$
\begin{aligned}
& =\chi\left(\phi\left(\max \left\{p\left(x_{n}, x_{n+1}\right), p\left(x_{n}, T x_{n}\right), p\left(x_{n+1}, F x_{n+1}\right), \frac{p\left(x_{n}, F x_{n+1}\right)+p\left(x_{n+1}, T x_{n}\right)}{2}\right\}\right)\right) \\
& =\chi\left(\phi\left(\max \left\{p\left(x_{n}, x_{n+1}\right), p\left(x_{n}, x_{n+1}\right), p\left(x_{n+1}, x_{n+2}\right), \frac{p\left(x_{n}, x_{n+2}\right)+p\left(x_{n+1}, x_{n+1}\right)}{2}\right\}\right)\right) .
\end{aligned}
$$

Since,

$\frac{p\left(x_{n}, x_{n+2}\right)+p\left(x_{n+1}, x_{n+1}\right)}{2}$

$$
\begin{aligned}
& \leq \frac{p\left(x_{n}, x_{n+1}\right)+p\left(x_{n+1}, x_{n+2}\right)-p\left(x_{n+1}, x_{n+1}\right)+p\left(x_{n+1}, x_{n+1}\right)}{2} \\
& =\frac{p\left(x_{n}, x_{n+1}\right)+p\left(x_{n+1}, x_{n+2}\right)}{2}
\end{aligned}
$$

then,

$$
\begin{aligned}
\phi\left(p\left(x_{n+1}, x_{n+2}\right)\right) & =\phi\left(p\left(T x_{n}, F x_{n+1}\right)\right) \\
& \leq \chi\left(\phi\left(\max \left\{p\left(x_{n}, x_{n+1}\right), p\left(x_{n+1}, x_{n+2}\right)\right\}\right)\right)
\end{aligned}
$$

If $\max \left\{p\left(x_{n}, x_{n+1}\right), p\left(x_{n+1}, x_{n+2}\right)\right\}=p\left(x_{n+1}, x_{n+2}\right)$, then,

$$
\left.\phi\left(p\left(x_{n+1}, x_{n+2}\right)\right)=\phi\left(p\left(T x_{n}, F x_{n+1}\right)\right) \leq \chi\left(\phi\left(p\left(x_{n+1}, x_{n+2}\right)\right\}\right)\right)<\phi\left(p\left(x_{n+1}, x_{n+2}\right)\right),
$$

which is a contradiction. Hence $\max \left\{p\left(x_{n}, x_{n+1}\right), p\left(x_{n+1}, x_{n+2}\right)\right\}=p\left(x_{n}, x_{n+1}\right)$. Thus,

$$
\left.\phi\left(p\left(x_{n+1}, x_{n+2}\right)\right)=\phi\left(p\left(T x_{n}, F x_{n+1}\right)\right) \leq \chi\left(\phi\left(p\left(x_{n}, x_{n+1}\right)\right\}\right)\right)<\phi\left(p\left(x_{n}, x_{n+1}\right)\right),
$$

which implies that the sequence $\left\{\phi\left(a_{n}\right)\right\}$ is a decreasing sequence in $[0, \infty)$, for all integers $n \geq 0$ and it is bounded below.

Let $a$ be the limit point of the sequence $\left\{\phi\left(a_{n}\right)\right\}$. It is obvious that $a \in[0, \infty)$.

We claim that $a=0$. In contrary suppose that $a>0$, applying $\limsup _{n \rightarrow \infty}$ in (2.22) we obtain,

$$
\begin{aligned}
0<a & \leq \limsup _{n \rightarrow \infty} \chi\left(\phi\left(a_{n}\right)\right) \\
& \leq \chi(a)<a
\end{aligned}
$$

which contradicts the assumption on the mapping $\chi$, hence $a=0$. Therefore, $\lim _{n \rightarrow \infty} \phi\left(a_{n}\right)=$ $\lim _{n \rightarrow \infty} \phi\left(p\left(x_{n}, x_{n+1}\right)\right)=0$.

It is clear that, $\lim _{n \rightarrow \infty} \phi\left(a_{n}\right)=\phi\left(\lim _{n \rightarrow \infty} a_{n}\right)=0$. From the definition of $\phi$ we see that,

$$
\lim _{n \rightarrow \infty} a_{n}=\lim _{n \rightarrow \infty} p\left(x_{n}, x_{n+1}\right)=0 .
$$

Now, we will show that a sequence $\left\{x_{n}\right\}$ is a Cauchy sequence in $X$. In contrary suppose that $\left\{x_{n}\right\}$ is not a Cauchy sequence. Therefore, there exists $\epsilon>0$ and a sequence of integers $m(k), n(k)$ such that,

$$
p\left(x_{n(k)}, x_{m(k)}\right) \geq \epsilon,
$$

for all $n(k)>m(k) \geq k$ for some $k \geq 0$.

Moreover, suppose that $m(k)$ is chosen as the smallest integer in such away that (2.25) holds so that we have,

$$
p\left(x_{(k)}, x_{m(k)-1}\right)<\epsilon
$$


Now, for all $n(k)>m(k)$ we have,

$$
\begin{aligned}
p\left(x_{n(k)}, x_{m(k)}\right) & \leq p\left(x_{n(k)}, x_{m(k)-1}\right)+p\left(x_{m(k)-1}, x_{m(k)}\right)-p\left(x_{m(k)-1}, x_{m(k)-1}\right) \\
& \leq p\left(x_{n(k)}, x_{m(k)-1}\right)+p\left(x_{m(k)-1}, x_{m(k)}\right) \\
& \leq p\left(x_{n(k)}, x_{m(k)-1}\right)+a_{n(k)} .
\end{aligned}
$$

As $k \rightarrow \infty$ in (2.27) and considering (2.24) we see that,

$$
p\left(x_{n(k)}, x_{m(k)}\right) \rightarrow \epsilon
$$

Similarly, by elementary computations we see that,

$$
p\left(x_{n(k)-1}, x_{m(k)-1}\right) \rightarrow \epsilon .
$$

From (2.18) we have,

$$
\begin{aligned}
\phi\left(p\left(x_{n(k)}, x_{m(k)}\right)\right) & =\phi\left(p\left(T x_{n(k)-1}, F x_{m(k)-1}\right)\right) \\
& \leq \chi\left(\phi\left(S\left(x_{n(k)-1}, x_{m(k)-1}\right)\right)\right)
\end{aligned}
$$

where,

$$
\begin{aligned}
S\left(x_{n(k)-1}, x_{m(k)-1}\right) & \\
= & \max \left\{p\left(x_{n(k)-1}, x_{m(k)-1}\right), p\left(x_{n(k)-1}, T x_{n(k)-1}\right), p\left(x_{m(k)-1}, F x_{m(k)-1}\right),\right. \\
& \left.\frac{p\left(x_{n(k)-1}, F x_{m(k)-1}\right)+p\left(x_{m(k)-1}, T x_{n(k)-1}\right)}{2}\right\} \\
= & \max \left\{p\left(x_{n(k)-1}, x_{m(k)-1}\right), p\left(x_{n(k)-1}, x_{n(k)}\right), p\left(x_{m(k)-1}, x_{m(k)}\right),\right. \\
& \left.\frac{p\left(x_{n(k)-1}, x_{m(k)}\right)+p\left(x_{m(k)-1}, x_{n(k)}\right)}{2}\right\},
\end{aligned}
$$

as $k \rightarrow \infty$ in (2.31) and considering (2.28) and (2.29), then (2.30) becomes,

$$
0<\phi(\epsilon) \leq \chi(\phi(\epsilon))<\phi(\epsilon) .
$$

Since $\phi(\epsilon) \in \overline{\phi(P)} \backslash\{0\}$ and $\chi(t)<t$, for all $t>0$, then (2.32) contains a contradiction. Therefore, the sequence $\left\{x_{n}\right\}$ is a Cauchy sequence and $\lim _{n, m \rightarrow \infty} p\left(x_{n}, x_{m}\right)=0$.

Since $X$ is complete, then we can choose a point $z \in X$ such that $\lim _{n \rightarrow \infty} p\left(x_{n}, z\right)=0$. Now, we prove that $z$ is a fixed point of $F$. In contrary suppose that $z \neq F z$.

From (2.18) we have,

$$
\begin{aligned}
\phi\left(p\left(x_{n+1}, F z\right)\right) & =\phi\left(p\left(T x_{n}, F z\right)\right) \\
& \leq \chi\left(\phi\left(S\left(x_{n}, z\right)\right)\right) .
\end{aligned}
$$

where,

$$
\begin{aligned}
S\left(x_{n}, z\right) & =\max \left\{p\left(x_{n}, z\right), p\left(x_{n}, T x_{n}\right), p(z, F z), \frac{p\left(x_{n}, F z\right)+p\left(z, T x_{n}\right)}{2}\right\} \\
& =\max \left\{p\left(x_{n}, z\right), p\left(x_{n}, x_{n+1}\right), p(z, F z), \frac{p\left(x_{n}, F z\right)+p\left(z, x_{n+1}\right)}{2}\right\}
\end{aligned}
$$


taking limit as $n \rightarrow \infty$ in (2.34) we get,

$$
S\left(x_{n}, z\right) \rightarrow p(z, F z)
$$

Applying limit as $n \rightarrow \infty$ in (2.33) we have,

$$
\phi(p(z, F z)) \leq \chi(\phi(p(z, F z)))<\phi(p(z, F z))
$$

which is a contradiction, hence $z=F z$.

Now, we shall show that a point $z$ is a unique common fixed of $T$ and $F$.

In contrary, suppose that $T$ and $F$ have two common fixed points $x_{0}$ and $y_{0}$ in $X$ with $x_{0} \neq y_{0}$ respectively. Thus, from Lemma 9 we have, $p\left(x_{0}, y_{0}\right)>0$.

From (2.18), for all $x_{0}, y_{0} \in X$ we have,

$$
\phi\left(p\left(T x_{0}, F y_{0}\right)\right)=\phi\left(p\left(x_{0}, y_{0}\right)\right) \leq \chi\left(\phi\left(S\left(x_{0}, y_{0}\right)\right)\right)
$$

where,

$$
\begin{aligned}
S\left(x_{0}, y_{0}\right)= & \max \left\{p\left(x_{0}, y_{0}\right), p\left(x_{0}, T x_{0}\right), p\left(y_{0}, F y_{0}\right), \frac{p\left(x_{0}, F y_{0}\right)+p\left(y_{0}, T x_{0}\right)}{2}\right\} \\
= & \max \left\{p\left(x_{0}, y_{0}\right), p\left(x_{0}, x_{0}\right), p\left(y_{0}, y_{0}\right), \frac{p\left(x_{0}, y_{0}\right)+p\left(y_{0}, x_{0}\right)}{2}\right\} \\
& =p\left(x_{0}, y_{0}\right) .
\end{aligned}
$$

Thus, (2.37) becomes,

$$
\phi\left(p\left(T x_{0}, F y_{0}\right)\right)=\phi\left(p\left(x_{0}, y_{0}\right)\right) \leq \chi\left(\phi\left(p\left(x_{0}, y_{0}\right)\right)\right)<\phi\left(p\left(x_{0}, y_{0}\right)\right) .
$$

which is a contradiction. Therefore, $T$ and $F$ have a unique common fixed point, that is $x_{0}=y_{0}$.

In contrary to the hypothesis of the above theorem, suppose that $x_{0}$ is a fixed point of $T$ and $x_{0} \neq F x_{0}$.

From (2.18), for all $x_{0} \in X$ we have,

$$
\phi\left(p\left(T x_{0}, F x_{0}\right)\right)=\phi\left(p\left(x_{0}, F x_{0}\right)\right) \leq \chi\left(\phi\left(S\left(x_{0}, F x_{0}\right)\right)\right)
$$

where,

$$
S\left(x_{0}, F x_{0}\right)
$$

$$
\begin{aligned}
& =\max \left\{p\left(x_{0}, F x_{0}\right), p\left(x_{0}, T x_{0}\right), p\left(F x_{0}, F^{2} x_{0}\right), \frac{p\left(x_{0}, F^{2} x_{0}\right)+p\left(F x_{0}, T x_{0}\right)}{2}\right\} \\
& =\max \left\{p\left(x_{0}, F x_{0}\right), p\left(x_{0}, x_{0}\right), p\left(F x_{0}, F^{2} x_{0}\right), \frac{p\left(x_{0}, F^{2} x_{0}\right)+p\left(F x_{0}, x_{0}\right)}{2}\right\} \\
& =p\left(x_{0}, F x_{0}\right) .
\end{aligned}
$$

Thus, (2.39) becomes,

$$
\phi\left(p\left(T x_{0}, F x_{0}\right)\right)=\phi\left(p\left(x_{0}, F x_{0}\right)\right) \leq \chi\left(\phi\left(p\left(x_{0}, F x_{0}\right)\right)\right)<\phi\left(p\left(x_{0}, F x_{0}\right)\right)
$$

which is a contradiction, hence $x_{0}=F x_{0}$. Similarly, it is easy to show that any fixed point of $F$ is also a fixed point of $T$. 


\section{References}

[1] M. Akkouchi, On a fixed point theorem of DW Boyd and JS Wong, Acta Math. Vietnam, 27 (2) (2002), 231-237.

[2] S. Banach, Sur les oprations dans les ensembles abstraits et leur application aux quations intgrales, Fund. Math., 3 (1) (1922), 133-181.

[3] D. W. Boyd and J. S. Wong, On nonlinear contractions, Proc. Amer. Math. Soc., 20 (2) (1969), 458-464.

[4] M. Bukatin, R. Kopperman, S. Matthews and H. Pajoohesh, Partial metric spaces, The American Mathematical Monthly, 116 (8), (2009), 708-718.

[5] M. Edelstein, On fixed and periodic points under contractive mappings, J. London Math. Soc., 1 (1) (1962), 74-79.

[6] Z. Kadelburg, H. K. Nashine and S. Radenovic, Fixed point results under various contractive conditions in partial metric spaces, Revista de la Real Academia de Ciencias Exactas, Fisicas y Naturales. Serie A. Matematicas, 107 (2) (2013), 241-256.

[7] Matthews, S., Partial metric topology in Papers on General Topology and Applications, Eighth Summer Conference at Queens College. Eds. S. Andima et al., Annals of the New York Academy of Sciences Vol. 728(1994), $183-197$.

[8] T. Nazir, and M. Abbas,Common fixed points of two pairs of mappings satisfying (EA)-property in partial metric spaces, Journal of Inequalities and Applications, 2014 (1) ( 2014), 237.

[9] E. Rakotch, A note on contractive mappings, Proc. Amer. Math. Soc., 13 (3) (1962), 459-465.

[10] S. Romaguera, Fixed point theorems for generalized contractions on partial metric spaces, Topology and its Applications, 159 (1) (2012), 194-199. 